\title{
Propiedades de aceros microaleados utilizados para el transporte de gas
}

\section{ácido}

\author{
E. Saldaña*, H. Castro*, C. Rodríguez* y J. Belzunce*
}

Resumen

El objetivo de este trabajo ha sido valorar el deterioro inducido por la entrada de hidrógeno en la microestructura y en las propiedades mecánicas de dos familias de aceros microaleados API (grados X-52 y X-65). La caracterización se ha realizado en estado bruto de laminación y tras someter al acero al ensayo NACE TM284/87, en un medio de $\mathrm{pH} 3,5$. Se ha evaluado la microestructura de los aceros, sus propiedades mecánicas y su comportamiento a fractura y, también, se ha cuantificado el agrietamiento inducido por la entrada de hidrógeno, la localización de estas grietas en relación con la geometría de la chapa y el modo como se propagan a través de la microestructura de los aceros.

Palabras clave Fragilización inducida por hidrógeno (FIH). Aceros microaleados. Propiedades mecánicas. Mecánica de la fractura.

\section{Properties of microalloyed steels used for sour gas transportation}

\begin{abstract}
The aim of this work was to evaluate the hydrogen induced demage on the microstructure and mechanical properties of API steels (X-52 and X-65 grades). Both steels were characterized in the as-rolled state and after being submitted to NACE TM284/87, pH 3.5, test. Microstructure, mechanical properties and fracture behaviour have been determined along with the internal cracking due to hydrogen. The appearence of these cracks were located in reference to the geometry of the plate and the way they propagate through the steel microstructures was aldo assessed.
\end{abstract}

Keywords Hydrogen induced cracking (HIC). Microalloyed steels. Mechanical properties. Fracture mechanics.

\section{INTRODUCCIÓN}

En la actualidad, los oleoductos y gaseoductos destinados al transporte de crudos con contenidos apreciables de gas ácido $\left(\mathrm{H}_{2} \mathrm{~S}\right)$ se construyen a partir de chapas de aceros microaleados. Estos materiales, se fabrican aplicando técnicas de laminación controlada, lo que permite obtener unos niveles de resistencia adecuada (grados API X-52, $\mathrm{X}-65$ y X-70), con alta tenacidad, buena soldabilidad y, especialmente, con unas microestructuras que ofrezcan la máxima resistencia a la fragilización por hidrógeno. A este último respecto, es bien conocido que la entrada de hidrógeno y su interacción con la microestructura del acero induce el deterioro de las propiedades mecánicas de los mis- mos. Este fenómeno, denominado fragilización inducida por el hidrógeno (FIH), se manifiesta de muy diversas formas, siendo cada manifestación FIH el resultado de complejas interacciones de múltiples variables, tales como la composición química, microestructura, contenido y distribución de inclusiones, prácticas de fabricación, dureza, grado de deformación, estado tensional, etc ${ }^{[1]}$.

En años recientes, la adopción, por parte de la industria, de aceros con un mayor grado de resistencia mecánica ha venido acompañada de un aumento de la susceptibilidad al FIH. Por esta razón, la comprensión de la interacción de los factores antes mencionados es de vital importancia para abordar el desarrollo de familias de aceros con una susceptibilidad al FIH reducida, que puedan ser

(*) E.T.S.I.I.G., Universidad de Oviedo, Campus Universitario, 33203 Gijón, Asturias (España). 
utilizados en este tipo de aplicaciones con la máxima fiabilidad.

\section{PARTE EXPERIMENTAL}

\subsection{Materiales}

Este trabajo ha sido realizado sobre dos muestras de acero API: uno de grado X-52 y otro de grado $\mathrm{X}$-65. Los materiales han sido caracterizados y ensayados en estado bruto de laminación y tras fragilización por hidrógeno en un medio NACE ácido. La tabla I muestra las composiciones químicas de los dos aceros. Nótese, principalmente, el ligero incremento en el contenido de carbono, manganeso y molibdeno del acero $3 \mathrm{~A}$ con objeto de lograr la mayor resistencia mecánica que exige el grado $\mathrm{X}-65$.

\subsection{Análisis metalográfico}

Se han utilizado técnicas de microscopía óptica y electrónica de barrido para determinar el tamaño medio del grano de ferrita (ASTM E112), la fracción volumétrica de perlita (ASTM E562), el espaciado interlaminar perlítico $y$, también, se ha procedido al recuento de inclusiones (ASTM E45). La probetas fueron pulidas hasta $1 \mu \mathrm{m}$ y atacadas con una solución de Nital al 3\%. Estos análisis se han realizado sobre una muestra tomada del centro de la chapa.

\subsection{Ensayo NACE TM284/87}

Este ensayo $^{[2]}$ consiste en la inmersión de las probetas en agua de mar sintética, libre de oxígeno (medio de ensayo NACE TM0177 $7^{[3]}$ ). Durante todo el ensayo se hace burbujear gas $\mathrm{H}_{2} \mathrm{~S}$, de manera que se alcanza la saturación y se mantiene un $\mathrm{pH}$ igual a 3,5 durante las $96 \mathrm{~h}$ que dura el ensayo. Se ensayaron 3 probetas de cada acero, extraídas de tres zonas diferentes de la chapa, correspondientes al centro, a la posición $1 / 4$ y al borde de la misma. Las probetas, una vez ensayadas, se examinan en busca de ampollas superficiales, siendo, posteriormente, seccionadas y pulidas metalográficamente. La superficies obtenidas se examinan en busca de grietas, determinándose los índices de susceptibilidad al hidrógeno, FIH, de acuerdo con las expresiones que se presentan a continuación.

$\mathrm{CTR}=\frac{\sum \mathrm{a}}{\mathrm{W}} \mathrm{CLR}=\frac{\sum \mathrm{b}}{\mathrm{T}} \mathrm{CSR}=\frac{\sum(\mathrm{a} \times \mathrm{b})}{\mathrm{W} \times \mathrm{T}} \mathrm{ETC}=\max (\mathrm{b})$

Dado que este tipo de ensayo está sujeto a una gran variabilidad ${ }^{[4]}$, el recuento del agrietamiento y el cálculo de los índices citados se ha realizado sobre 9 secciones por probeta (la norma NACE exige realizar el recuento sobre 3 secciones por probeta), extraídas deposiciones situadas en el borde, un cuarto del ancho y centro de la chapa. Así mismó, se decidió evaluar la situación de las grietas, con relación al espesor de cada probeta, mostrándose el resultado en la figura 1 , donde

a es la longitud de la grieta,

b es el espesor de la grieta,

W es el ancho de la sección y

$\mathrm{T}$ es el espesor de la chapa.

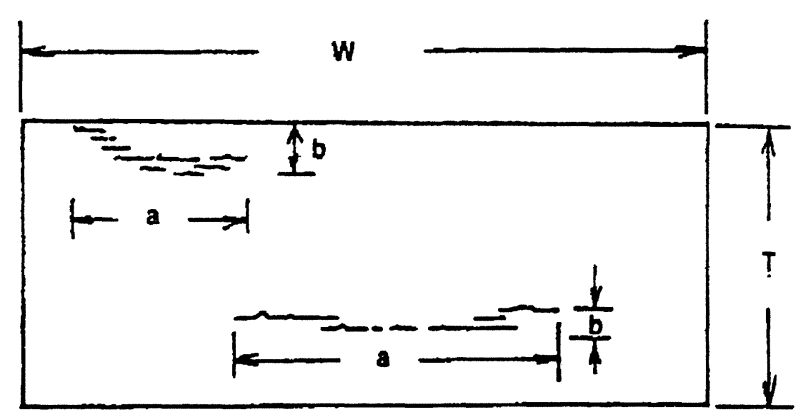

Figura 1. Evaluación de la fisuración inducida por el hidrógeno.

Figure 1. Hydrogen induced cracking evaluation.

Tabla I. Composición química de los aceros

Table I. Chemical analysis of the steels

\begin{tabular}{|c|c|c|c|c|c|c|c|c|c|c|c|c|c|c|c|c|}
\hline Material & $C$ & $\mathrm{Si}$ & $M n$ & $\mathrm{Cr}$ & $\mathrm{Ni}$ & $\mathrm{Cu}$ & $\mathrm{Ti}$ & $\mathbf{V}$ & Mo & $\mathrm{Nb}$ & Sn & $P$ & $S$ & Al & As & $\mathbf{N}$ \\
\hline $3 A \times 65$ & 0,066 & 0,285 & 10,31 & 0,044 & 0,022 & 0,320 & 0,006 & 0,065 & 0,082 & 0,047 & 0,002 & 0,011 & 0,002 & 0,036 & 0,002 & 0,0061 \\
\hline $9 A \times 52$ & 0,061 & 0,236 & 10,05 & 0,020 & 0,012 & 0,257 & 0,011 & 0,049 & 0,002 & 0,042 & 0,006 & 0,014 & 0,001 & 0,033 & 0,001 & 0,0055 \\
\hline
\end{tabular}


E. Saldaña, H. Castro, C. Rodríguez y J. Belzunce

\subsection{Ensayos mecánicos}

Se ejecutaron ensayos de dureza Vickers, con carga de $30 \mathrm{~kg}$ y ensayos de tracción sobre probeta cilíndrica, de acuerdo con la norma UNE 7-474-92, bajo una velocidad de $5 \mathrm{~mm} / \mathrm{min}$ y ensayos de fractura, con objeto de determinar las curvas de resistencia (curvas J-R), sobre probeta compacta (tipo CT), con un tamaño de grieta relativo al ancho de la probeta, $a / W=0,6$, de acuerdo con la norma ESIS P2-92. Se utilizó una velocidad de ensayo de $5 \cdot 10^{-6} \mathrm{~m} / \mathrm{s}$. Todos estos ensayos se han realizado a temperatura ambiente, en dos condiciones: en estado bruto de laminación, al aire, y, tras someter las probetas al ensayo NACE, durante $96 \mathrm{~h}$.

\section{RESULTADOS Y DISCUSIÓN}

La tabla II muestra los resultados del análisis metalográfico realizado sobre ambos aceros. La microestructura de los dos aceros era ferrito-perlítica de grano fino y, también, se observaba con nitidez una cierta segregación central, especialmente en el caso del acero 3A. En ambos casos, se había procedido a la inyección de calcio antes de la colada del acero, con el propósito de modificar la forma de las inclusiones. El contenido inclusionario es muy bajo, en virtud del bajo contenido final de azufre de los dos aceros.

La tabla III muestra el valor del parámetro CLR, evaluado en los dos aceros, en las probetas extraídas del centro, $1 / 4$ y borde de las chapas. La figura 2 muestra el aspecto característico de las grietas de hidrógeno inducidas en este ensayo. Se hace notar que estas grietas se generan en ausencia de tensiones aplicadas, es decir, son debidas a la propia presión interna local que genera el hidrógeno al acumularse en determinadas regiones específicas de la microestructura del acero. Se pone de manifiesto que las grietas tienden a propagarse, de manera escalonada, a través de las colonias de perlíta. La figura 3 muestra el tamaño y la situación,
Tabla III. Resultados del ensayo NACE TM284/90, pH 3.5

Table III. Results of NACE TM284/90, pH 3.5, test

\begin{tabular}{cccc}
\hline Aceros & $\begin{array}{c}\text { CLR } \\
\text { (centro) }\end{array}$ & $\begin{array}{c}\text { CLR } \\
\text { (posición 1/4) }\end{array}$ & $\begin{array}{c}\text { CLR } \\
\text { (borde) }\end{array}$ \\
\hline 3 A X65 & 17,6 & 12,4 & 4,2 \\
9 A X52 & 0,3 & 0,2 & 2,4 \\
\hline
\end{tabular}

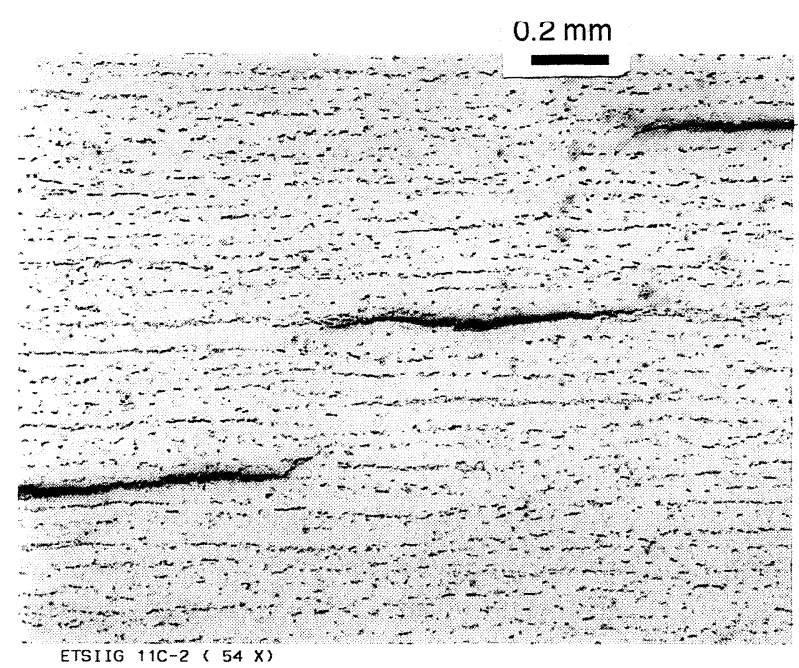

Figura 2. Fisuración inducida por el hidrógeno en el ensayo NACE (acero 3A, Centro).

Figure 2. HIC after NACE test (steel 3A, center).

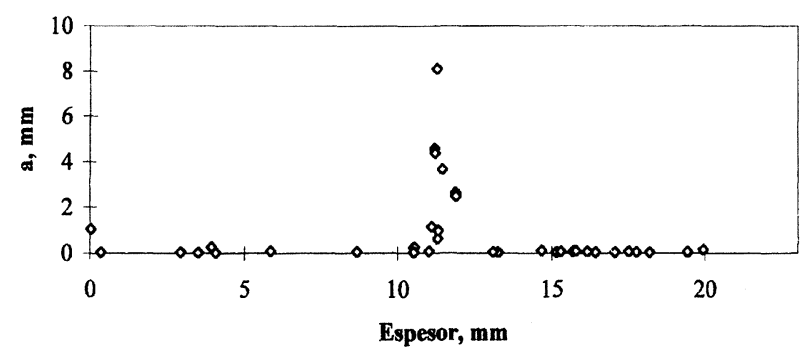

Figura 3. Localización de la situación de las grietas en relación al espesor (acero 3A, centro).

Figure 3. Crack's localization along the thickness of coupons (steel 3A, center).

Tabla II. Parámetros metalográficos

Table II. Metalographics parameters

\begin{tabular}{cccccc}
\hline Material & $\begin{array}{c}\text { Tamaño de grano } \\
\text { ASTM }\end{array}$ & $\begin{array}{c}\text { Fracción volumétrica } \\
\text { de perlita (\%) }\end{array}$ & $\begin{array}{c}\text { Fracción volumétrica } \\
\text { de inclusiones }(\%)\end{array}$ & Morfología & Tipo \\
\hline 3A X65 & 9,5 & 4,5 & 0,0734 & Esférica & $(\mathrm{Al}, \mathrm{Ca}, \mathrm{Mg}) \mathrm{S},(\mathrm{Ca}, \mathrm{Al}) \mathrm{O}$ \\
$9 \mathrm{~A} \times 52$ & 9,6 & 5 & 0,0560 & Esférica & $\mathrm{CaS},(\mathrm{Ca}, \mathrm{Al}) \mathrm{O},(\mathrm{Ca}, \mathrm{Mg}) \mathrm{S}$ \\
\hline
\end{tabular}


en relación al espesor de las chapas, de las grietas individuales detectadas en secciones específicas. Se pone de manifiesto que el acero $3 \mathrm{~A}$ presenta un agrietamiento localizado en el centro del espesor de la chapa, es decir, está ligado a la segregación central, lo que, también, justifica el inferior índice CLR determinado en la probeta del borde ${ }^{[5 \text { y } 6]}$ (Tabla II).

La tabla IV muestra los resultados de los ensayos de dureza, tracción (límite elástico, resistencia mecánica, alargamiento y estricción) y fractura $\left(\mathrm{J}_{\mathrm{Ic}}\right.$, valor de $\mathrm{J}$ para el inicio de la propagación estable de la grieta) realizados en las dos condiciones de ensayo: al aire y tras $96 \mathrm{~h}$ de exposición en el medio NACE de pH 3,5. La resistencia mecánica de los aceros no se modifica sensiblemente con la inmersión en el medio fragilizante, a pesar de que, en el caso del acero $3 \mathrm{~A}$, se puso de manifiesto la existencia de una grieta central claramente visible a simple vista en la sección de fractura, asociada a la segregación presente en estas chapas. Por otro lado, el acero 9A tiene una resistencia mecánica inferior y una tenacidad apreciablemente mayor que las del acero $3 \mathrm{~A}$, y esa es la razón que justifica los menores índices de agrietamiento que se habían registrado en el ensayo NACE (Tabla II).

La figura 4 expone las curvas $\mathrm{J}-\mathrm{R}$ obtenidas con ambos aceros en estado bruto de laminación (ensayos al aire) y tras inmersión en el medio NACE. Es especialmente interesante destacar que la inmersión durante 96 h en el medio NACE, a pesar de inducir un agrietamiento interno que, en el caso del acero 3A, es importante, no modifica sensiblemente el comportamiento general a fractura ni los parámetros específicos de fractura característicos de este acero. En el caso del acero 9A que, de entre los dos, era el acero menos sensible a la fragilización por hidrógeno, la inmersión en el medio NACE ha provocado una cierta pérdida de tenacidad (parámetro $\mathrm{J}_{\text {Ic }}$ y curva J-R) aunque, de cualquier manera, ésta sigue siendo superior a la del acero $3 \mathrm{~A}$.

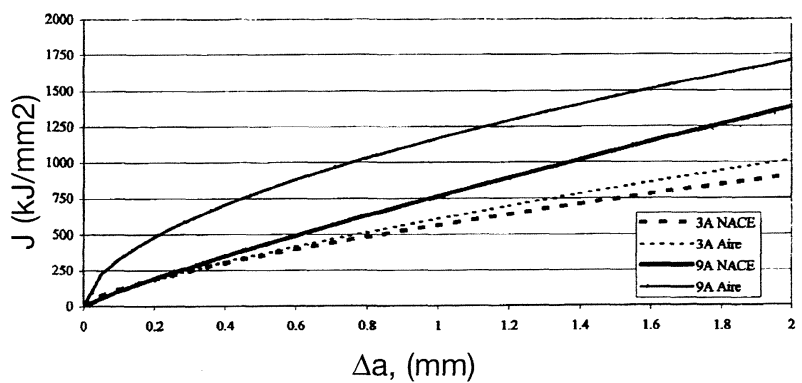

Figura 4. Curvas J-R de los aceros en ensayos al aire y tras exposición en el medio NACE.

Figure 4. J-R curves of tested coupons, as rolled and after NACE conditions.

\section{CONCLUSIONES}

Mientras que, en los aceros de resistencia media (API X-52), el control inclusionario y microestructural (grano fino) permiten obtener aceros de alta tenacidad con una reducida susceptibilidad al agrietamiento por hidrógeno, el incremento de aleantes (especialmente $\mathrm{Mn}$ ) que es preciso introducir en el caso de los aceros más resistentes (API X-65), promueve la segregación central y, estas regiones, son ya susceptibles al agrietamiento por hidrógeno.

Por otro lado, se ha puesto de manifiesto que, ni la presencia de hidrógeno atrapado en el interior de la microestructura ni siquiera la existencia de grietas internas afectan, de una manera importante, a las propiedades mecánicas y a los parámetros de fractura de los aceros estudiados, al menos en la gama de velocidades de aplicación de carga utilizada en esta experimentación $\left(5 \cdot 10^{-6} \mathrm{~m} / \mathrm{s}\right)$.

\section{Agradecimientos}

Este trabajo ha sido financiado por la Dirección General de Enseñanza Superior e Investigación Científica (Proyecto FEDER,1FD97-0638-C02),

Tabla IV. Resultados de ensayos mecánicos y de fractura

Table IV. Results of mechanical and fracture tests

\begin{tabular}{cccccccc}
\hline Material & HV & Medio & $\sigma_{\text {YS }}(\mathbf{M P a})$ & $\sigma_{U}(\mathbf{M P a})$ & $\mathbf{A}(\%)$ & $\mathbf{Z}(\%)$ & $\mathbf{J}_{\mathbf{I c}}\left(\mathbf{k J} / \mathbf{m}^{2}\right)$ \\
\hline \multirow{2}{*}{ 3A X65 } & 155 & Aire & 450 & 530 & 31,7 & 76 & 189 \\
& & NACE pH 3,5 & 427 & 514 & 28,7 & 45,5 & 190,2 \\
9A X52 & \multirow{2}{*}{146} & Aire & 391 & 475 & 38,3 & 75 & 496 \\
& & NACE pH 3,5 & 410 & 479 & 31,4 & - & 223,4 \\
\hline
\end{tabular}


así como por el programa de becas MUTIS, AECI. Los autores agradecen, también, la colaboración de la empresa ACERALIA S.A., que ha suministrado los aceros empleados en este trabajo.

\section{REFERENCIAS}

[1] V. Schwinn y A. Stresselberg, Petroleum Production: Stepwise or Hydrogen Induced Cracking in Oil and Gas Production, NACE International, Houston, Estados Unidos, 1999, pp. 18-39.

[2] NACE TM0284/87: Test Method: Evaluation of Pipeline Steels for Resistance to Stepwise Cracking, NACE, Houston, Estados Unidos, 1987.
[3] NACE TM 01-77: Test Method: Testing of Metals for Resistance to SulfideStress Cracking at Ambient Temperature, NACE, Houston, Estados Unidos, 1977.

[4] C.J. BENNET y A. BRown, Wet $\mathrm{H}_{2} \mathrm{~S}$ Cracking of Carbon Steels and Weldments, NACE International, Houston, Estados Unidos, 1996, pp. 773-801.

[5] K. VAN GELDER et al., Wet $\mathrm{H}_{2} \mathrm{~S}$ Cracking of Carbon Steels and Weldments, NACE International, Houston, Estados Unidos, 1996, pp. 861-874.

[6] M. Elboujdaini y et al., Petroleum Production: Stepwise or Hydrogen Induced Cracking in Oil and Gas Production, NACE International, Houston, Estados Unidos, 1999, pp. 1-17. 